\title{
Elastolytic activity of sputum and its relation to purulence and to lung function in patients with bronchiectasis
}

\author{
RA STOCKLEY, SL HILL, HM MORRISON, CM STARKIE \\ From the General Hospital and the Department of Pathology, Selly Oak Hospital, Birmingham
}

\begin{abstract}
Sputum samples from 34 patients with bronchiectasis were assessed subjectively and the results related to objective measurements of elastase activity and albumin content. The results suggest that the macroscopic appearance of the sample is related to the elastase content, $88.7 \%$ of the purulent samples but none of the mucoid samples showing elastase activity. The macroscopic appearance was also associated with changes in protein transudation into the secretions. The median sputum:serum albumin concentration ratio was $0.71 \times 10^{-2}$ (range $0.22-4.7)$ in the mucoid samples but was greater in purulent samples $(\mathrm{p}<0.005)$, with a median value of $1.52 \times 10^{-2}$ (range $0.55-12.72$ ), suggesting that purulence in the stable state was associated with low grade pulmonary inflammation or epithelial damage or both. Abnormalities of air flow were found in 24 of the patients $(70.6 \%)$ but there was a significantly higher ratio of residual volume to total lung capacity $(\mathrm{p}<0.025)$ in patients who regularly produced purulent sputum (mean (SD) RV/TLC $44.4 \%(9.0 \%)$ ) than in those with mucoid or mucopurulent secretions $(38.0 \%(9.9 \%))$. A similar difference was found between those who produced elastase positive secretions and those who produced elastase negative ones.
\end{abstract}

The presence of active proteolytic enzymes in lung secretions has been implicated in the pathogenesis of several lung diseases, including the adult respiratory distress syndrome, cystic fibrosis, fibrosing alveolitis, and emphysema. ${ }^{1}$ Although the relevance of these enzymes to diseases of the lung connective tissue and interstitial space is uncertain, their relationship to pathological changes in the bronchial epithelium directly exposed to the secretions may be more precise. It has been shown that purified elastase from the neutrophil can cause damage to the bronchial epithelium and reduce ciliary function. ${ }^{2}$ Such proteolytic activity may be important in the pathogenesis of bronchiectasis, where abnormalities of the bronchial epithelium ${ }^{3}$ and reduced mucociliary function ${ }^{4}$ are features of the disease.

Previous studies from our laboratory have shown that elastase activity is not a feature of the secretions from patients with stable chronic obstructive bronchitis. $^{s}$ During acute exacerbations due to bacterial infection, however, free elastolytic activity is often

Address for reprint requests: Dr RA Stockley, General Hospital, Birmingham B4 6NH.

Accepted 23 January 1984 found. ${ }^{5}$ Subsequent studies of the secretions from patients with cystic fibrosis (which are often purulent in the stable clinical state) have shown elastase activity to be frequently present and probably derived from the neutrophil. ${ }^{6}$ Similarly, preliminary studies in patients with bronchiectasis have shown elastase activity to be present in some cases and thus potentially relevant to the pathogenesis of the disease.?

The present study was designed to investigate further these preliminary data and to determine whether free elastase activity is a regular feature of secretions obtained from patients with bronchiectasis and whether it is related to the type of secretion produced. The data obtained were intended to form the basis of subsequent studies on the relationship of the secretions to the progression of the disease and the results of therapeutic modification of the nature of the secretions.

\section{Methods}

Thirty four patients with a radiographic or biopsy diagnosis of bronchiectasis were studied over several months. The patients ( 20 of them women) ranged in 
age from 30 to 71 years (median 51). All had regular cough with sputum production. The patients were studied at least once in the stable clinical state, but samples were collected from most of the patients on subsequent occasions both during and after clinical exacerbations of their respiratory disease for different parts of the study. Twenty one had never smoked, nine were ex-smokers (not having smoked for at least two years), and four were current smokers. Nine patients had had lobectomy performed for bronchiectasis and one patient had had a thoracoplasty for tuberculosis. None had serum immunoglobulin deficiency.

The patients collected sputum samples into sterile plastic containers during four hours in the morning after rising. A portion of each sample was taken before centrifugation for bacteriological or microscopic analysis as indicated. The samples were ultracentrifuged at $50000 \mathrm{~g}$ for 90 minutes $\left(4^{\circ} \mathrm{C}\right)$ to obtain the sol phase, which was aspirated and stored at $-70^{\circ} \mathrm{C}$ until it was analysed (the remaining pellet was discarded).

A venous blood sample was obtained during the period when some of the sputum collections were made, allowed to clot, and centrifuged to obtain the serum. This was also stored at $-70^{\circ} \mathrm{C}$ until it was analysed.

All sputum samples were assessed in both a subjective and an objective manner. Firstly, all 198 samples obtained were assessed macroscopically and designated either as mucoid (colourless), mucopurulent (pale yellow), or purulent (yellow to green). A portion was then spread on a glass slide with a wooden spatula; it was then fixed and stained with haematoxylin and eosin $(n=201)$. These slides were then assessed independently on two occasions three months apart on a single blind basis. The presence of neutrophils was noted on each occasion; they were classified as either sparse or absent $(0 / \pm)$, present $(+/+)$, or numerous $(+++)$.

The samples were subsequently analysed more objectively in two ways. Firstly, the presence and degree of elastolytic activity was assessed by means of the elastin agarose diffusion plate. In brief, fine particulate elastin (bovine ligamentum nuchae) was obtained from P-L Biochemicals, Milwaukee, and suspended in molten agarose $(1 \% \mathrm{w} / \mathrm{v}$ in Barbitone buffer-0.05 mol/l, pH 8.6). The suspension was poured $1 \mathrm{~mm}$ thick on glass plates and allowed to set. The sputum sol phase samples were placed in wells cut in the gel and incubated for 48 hours with known concentrations of porcine pancreatic elastase (British Drug Houses). The clear areas of elastolysis were measured and compared with the standards of porcine pancreatic elastase (PPE); the measurements were expressed as the equivalent of
PPE/100 $\mu$ l sample (lower limit of measurement $0 \cdot 8$ $\mu \mathrm{g} / 100 \mu \mathrm{l}$; between batch coefficient of variation $4 \cdot 1 \%$ ). Where elastase was detectable but unmeasurable or absent its value was taken as zero for statistical purposes. Secondly, the albumin concentration was measured in both the sputum and the serum samples by rocket immunoelectrophoresis and the sputum:serum concentration ratio was obtained and multiplied by 100 for convenience. ${ }^{8}$ The forced expired volume in 1 second $\left(\mathrm{FEV}_{1}\right)$ and forced vital capacity (FVC) were measured in all of the patients. Two were unable to complete the tests but the remainder underwent assessment of total lung capacity (TLC) by helium dilution, residual volume (RV), and single breath transfer factor for carbon monoxide corrected for lung volume ( $\mathrm{Kco}$ ).

Differences in the elastase and albumin content of the macroscopically grouped samples were determined statistically with the Wilcoxon rank sum test (single tailed). A similar test was applied to the indices of lung function.

\section{Results}

One hundred and ninety eight individual sputum samples were assessed macroscopically for the degree of purulence. Fifty eight $(29.3 \%)$ were classified as mucoid, $51(25.8 \%)$ as mucopurulent, and $89(44.9 \%)$ as purulent. The results of the two assessments by microscopy are shown in table 1 . For most there was good agreement between the two attempts, the same category being used for the two assessments in $72-80 \%$ of cases.

The relationship betwen the first assessment by microscopy and the macroscopic appearance of the sputum is shown in table 2 . There is little relationship between the results of the two methods of analysis.

Elastase activity was measured in 132 of the samples studied by microscopy. The results are summarised in figure 1 . Elastase activity was found to be distributed throughout all three groups. Eight of the 41 samples $(19.5 \%)$ grouped $0 / \pm, 27$ of the 56

Table 1 Comparison of the microscopic grading of secretions on the basis of neutrophils* on two separate occasions (percentage agreement between the two attempts in parentheses)

\begin{tabular}{llll}
\hline Second grading & \multicolumn{3}{l}{ First grading } \\
\cline { 2 - 4 } & +++ & $+l++$ & $0 / \pm$ \\
\hline+++ & $39(72 \%)$ & 15 & 0 \\
$+/++$ & 9 & $51(76 \%)$ & 7 \\
$0 / \pm$ & 1 & 15 & $64(80 \%)$ \\
\hline $0 / \pm$ indicates & sparse or absent, $+/++$ present, and +++ \\
numerous. & &
\end{tabular}


Table 2 Comparison of the microscopic grading on the basis of neutrophils* with the macroscopic apearance of the secretions at collection (percentage showing free elastase activity in parentheses)

\begin{tabular}{lcll}
\hline $\begin{array}{l}\text { Microscopic } \\
\text { grading }\end{array}$ & \multicolumn{3}{l}{ Subjective assessment } \\
\cline { 2 - 4 } & Mucoid & Mucopurulent & Purulent \\
\hline+++ & 1 & 16 & $38(89 \%)$ \\
$+/++$ & 22 & $22(31 \%)$ & $35(88 \%)$ \\
$0 / \pm$ & 35 & $13(7 \%)$ & $16(62 \%)$ \\
\hline
\end{tabular}

*See table 1 for grading.

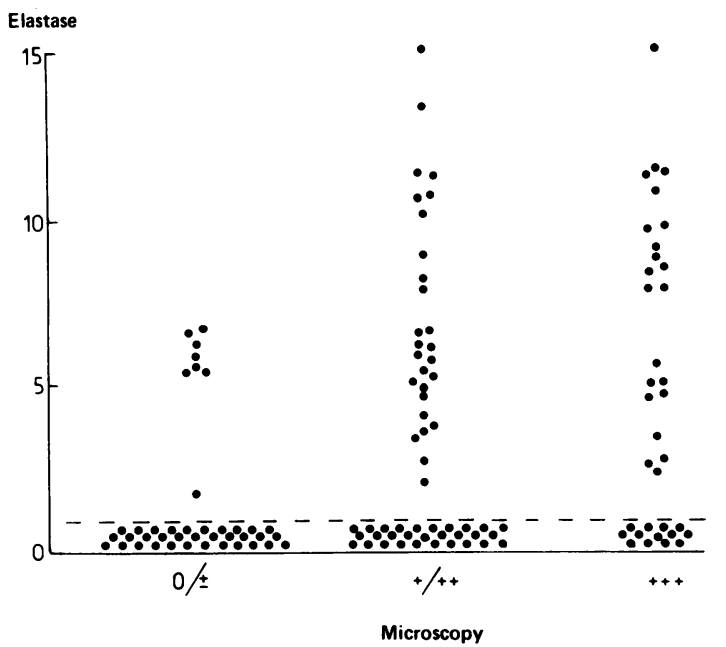

Fig 1 Elastase activity ( $\mu g$ porcine pancreatic elastase activity/100 $\mu$ l sample) related to microscopy grades $(0 / \pm$ indicates neutrophils sparse or absent, $+1++$ present, and +++ numerous). Each point is the result from one sample and the dotted line indicates the lower limit of detection.

$+/++$ samples $(48.2 \%)$ and 22 of the $35+++$ samples $(62.9 \%)$ were found to have detectable elastase activity.

A clearer relationship was found between the presence of elastase activity and the macroscopic assessment of the sputum. The results are summarised for 121 samples in figure 2 . None of the 29 mucoid samples showed elastase activity, whereas $20 \%$ of the 30 mucopurulent samples and $88.7 \%$ of the 62 purulent samples did.

There was no relationship between the sputum: serum albumin ratio and the microscopic assessment of the sputum. A progressive increase in the ratio was, however, seen with an increase in the degree of purulence of the secretions (fig 3). The ratio in 42 purulent samples where this was measured (median $1.52 \times 10^{-2}$, range $0.55-12.72$ ) was higher than in 25 mucopurulent samples (median $1.19 \times 10^{-2}$, range $=0 \cdot 19-6 \cdot 59)$. These latter values were in turn higher than those obtained in 28 mucoid samples (median $0 \cdot 71$, range $0 \cdot 22-4 \cdot 7$ ).

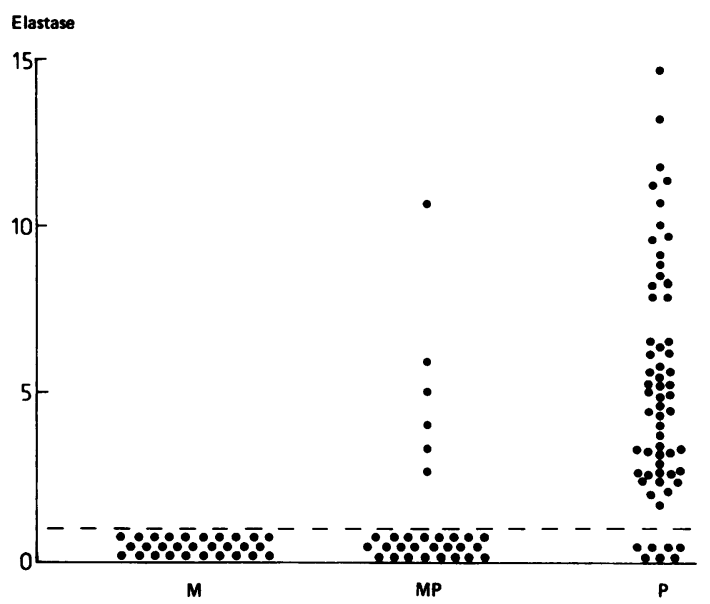

Fig 2 Elastase activity ( $\mu \mathrm{g}$ porcine pancreatic elastase acitivity/100 $\mu \mathrm{l}$ sample) related to macroscopic appearance of sputum. $M$-mucoid; $M P$-mucopurulent; $P$-purulent.

Examination of single sputum samples from 34 outpatients with bronchiectasis who were regarded as being well on clinical grounds showed $14.3 \%$ to be mucoid, $25.7 \%$ mucopurulent, and $60 \%$ purulent. Elastase activity was detectable in 21 $(61.8 \%)$ of the patients and ranged from 2.0 to 11.5 $\mu \mathrm{g}$ PPE $/ 100 \mu \mathrm{l}$. A similar wide range was seen in the sputum : serum albumin ratios of the patients

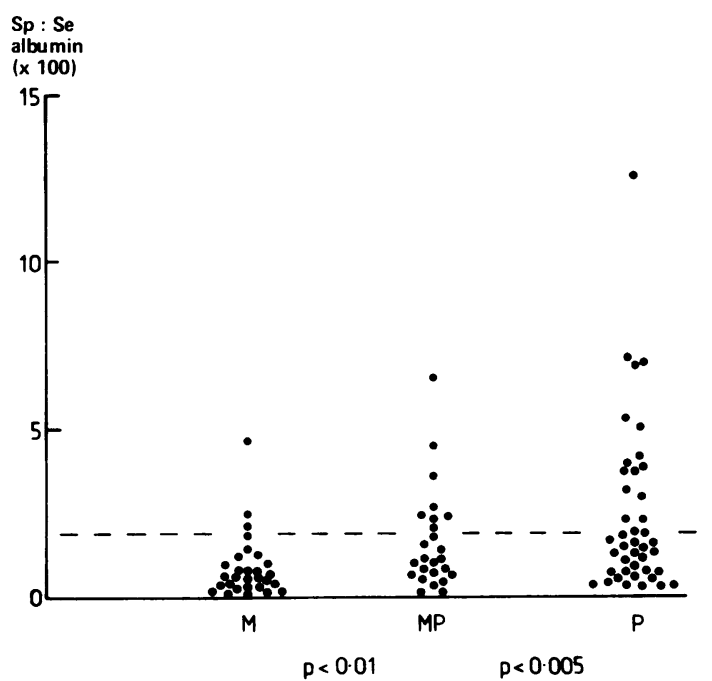

Fig 3 Sputum : serum albumin concentration ratio for those samples in each of the macroscopically defined groups in which it was determined. Each point represents one sample. The significance values for the difference between groups $M$ (mucoid) and MP (mucopurulent) and MP and $P$ (purulent) are shown. The dotted line represents the upper limit found in patients with stable obstructive bronchitis. ${ }^{5}$ 
Table 3 Average lung function values for all patients, those who regularly produce purulent secretions and those with mucoid and with mucopurulent secretions, and the elastase positive and negative groups

\begin{tabular}{|c|c|c|c|}
\hline & \multicolumn{3}{|c|}{ Mean (SD) lung function values* } \\
\hline & $F E V_{1} / F V C(\%)$ & $R V / T L C(\%)$ & $K C O\left(\operatorname{mmol}_{\min }-1 \mathrm{kPa}^{-1} 1^{-1}\right)$ \\
\hline $\begin{array}{l}\text { All patients } \\
\text { Purulent } \\
\text { Mucoid and mucopurulent } \\
\text { Elastase positive } \\
\text { Elastase negative }\end{array}$ & $\begin{array}{l}56 \cdot 8(15 \cdot 6) \\
54 \cdot 6(14 \cdot 5) \\
60 \cdot 0(17 \cdot 0) \\
n=20 \\
53 \cdot 9(14 \cdot 5) \\
61 \cdot 5(16 \cdot 7) \\
n=21 \\
n=13\end{array}$ & $\begin{array}{l}41 \cdot 8(9 \cdot 8) n=32 \\
44 \cdot 4(9 \cdot 0) n=19 \\
38.0(9 \cdot 9) n=13 \\
44 \cdot 7(8 \cdot 9) n=20 \\
36.9(9 \cdot 5) n=12\end{array}$ & 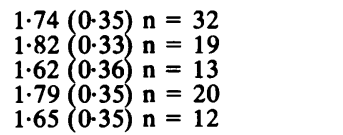 \\
\hline
\end{tabular}

*The forced expired volume in one second (FEV) is expressed as a percentage of the forced vital capacity (FVC) and the residual volume (RV) as a percentage of total lung capacity (TLC). The transfer factor for carbon monoxide (single breath) is shown corrected for volume (KCO).

(median $1.81 \times 10^{-2}$, range $0.59-11 \cdot 03$ ). The results of routine lung function testing are shown in table 3. Twenty four of the patients had evidence of airflow obstruction $\left(\mathrm{FEV}_{\mathrm{l}} / \mathrm{FVC}\right.$ ratio $\left.<65 \%\right)$ but no clear difference was found between those who produced purulent secretions regularly $(n=20)$ and the others. Two patients (one with purulent secretions) were unable to perform the further tests of pulmonary function. In the remainder there was a significant difference between the groups with purulent and mucoid-mucopurulent secretions for residual volume expressed as a percentage of total lung capacity (table $3 ; p<0.025$ ). The difference remained $(p<0.05)$ when the results were expressed as proportions of predicted normal values. ${ }^{9}$ The average values were $126.4 \%$ (SD $17.4 \%$ ) of the predicted values for the group with purulent secretions and $112.7 \%(23.7 \%)$ for the remainder.

The findings were similar when the patients were divided into those with elastase positive and those with elastase negative secretions. Most results were similar in the two groups but RV/TLC ratios (table 3) were higher for the elastase positive group ( $<<$ 0.01 ). Similarly, the RV/TLC ratios expressed as percentages of the predicted values were also greater $(\mathrm{p}<0.025)$ in the elastase positive group (mean (SD) $127.5 \%(18.0 \%)$ ) than in the elastase negative group $(109.6 \%(21.9 \%))$.

\section{Discussion}

Bronchiectasis is characterised by excess bronchial mucus and dilated bronchi, with loss of cartilage and elastic tissue and the presence of fibrosis. ${ }^{3}$ The epithelial lining often shows a loss of ciliated columnar cells and the presence of inflammatory cells and it occasionally shows ulceration, and mucociliary clearance is reduced. ${ }^{4}$ The pathogenesis and the effects of such changes have received little attention.

Proteolytic enzymes have been implicated in the pathogenesis of several chronic lung diseases, and leucocyte elastase has been shown to cause damage to bronchial epithelium and reduce ciliary function in vitro. ${ }^{2}$ There are, however, no data relating the character of lung secretions in bronchiectasis to their enzyme content.

The assessment of sputum remains largely subjective, mainly because sputum is a mixture of secretions from different regions of the bronchial tree variably contaminated with saliva and nasopharyngeal secretions. The difficulties were confirmed by the early studies, in which the neutrophil content of sputum smears was assessed by microscopy. Not only were the neutrophil numbers widely different in different fields of the same slide but when a simple assessment of the presence of neutrophils on each slide was repeated on a subsequent occasion by the same observer there was substantial disparity between the results (table 1). Furthermore, there was little agreement between these results and the macroscopic degree of purulence (table 2).

We found no relationship between the elastase content and the results of microscopic assessment. On the other hand, there seemed to be a relationship between elastase content and the macroscopic assessment. Elastase activity was not found in the mucoid samples, whereas it was an almost constant feature of purulent secretions. In this respect these secretions are similar to those of patients with bronchitis, ${ }^{5}$ which contain elastase activity only during acute infective episodes, and patients with cystic fibrosis. ${ }^{6}$ Our results support those of studies by Lieberman and Kaneshiro ${ }^{10}$ and Twumasi and Liener ${ }^{11}$ demonstrating that elastase activity is a feature of purulent sputum despite the subjective nature of the macroscopic appearance.

The presence of elastase activity is of interest not only because it may account for the reduced mucociliary function in these patients but also because it is potentially harmful to the epithelial cells themselves. In this respect the albumin results are of interest. We have shown previously that the measurement of albumin immunologically is not affected by the presence of elastase but that an increase in the sputum : serum albumin concen- 
tration ratio is a feaure of the inflammatory response to infection. ${ }^{58}$ In these samples, however, the sputum : serum albumin ratios showed a significant increase that was associated with the degree of purulence. This finding was not confined to samples obtained from patients during an exacerbation but was also a feature of the secretions obtained when patients were assessed as being well on clinical grounds. The results are similar to those obtained in patients with cystic fibrosis, where the sputum: serum albumin ratios are the same during an exacerbation as in the apparently well clinical state,$^{6}$ and unlike the ratios in patients with bronchitis, where a rise occurs only during exacerbations. Since albumin enters the secretions by diffusion, the high concentrations found in patients with purulent secretions suggest either a degree of constant inflammation in the lung or damage to the integrity of the epithelium. Whether this represents cause or effect is uncertain-though, in view of the in vitro effect of elastase on bronchial epithelium, ${ }^{2}$ it is tempting to suggest that the albumin leak is a direct consequence of the type of secretion produced by these patients. This hypothesis could be tested by therapeutic manipulation of the character of the secretions in the manner adopted in our recent studies on chronic bronchitis. ${ }^{12}$

The effect of purulent secretions on lung pathology and function in vivo is difficult to ascertain. Previous studies by Cherniak and Carton ${ }^{13}$ have suggested that lung function is related to the number of segments affected. Furthermore, the presence of airflow obstruction and air trapping were said to be features of the worst affected patients, although the nature of their secretions was not described. Subsequent studies ${ }^{14}$ have suggested that normal or moderately impaired airflow with some reduction in carbon monoxide transfer factor are features of the disease. Clinical impression suggests that the worst patients tend to have purulent secretions.

Interpretation of results of lung function measurement is complicated by the fact that several patients had undergone operative procedures that affect absolute lung volumes. For this reason only results for FEV $/ F V C, R V / T L C$, and transfer factor corrected for lung volume were considered. The transfer factor was essentially normal for all patients (perhaps because it had been corrected for volume, unlike values in previous studies. ${ }^{14}$ ) Several patients had airflow obstruction and, particularly in patients with purulent secretions, an increased RV/TLC ratio was observed. This was not clearly related to previous smoking history since only five of the 13 current ex-smokers produced purulent or elastase positive secretions, although with such small numbers this possibility cannot be ruled out. Interes- tingly, this difference in lung function between patients with different subjectively assessed types of secretion is also confirmed by objective assessment (table 3 ). The presence of elastase activity (potentially a damaging factor) is associated with the presence of more air trapping. Whether this represents a true causal effect is uncertain. The role of the type of secretion in the modification of lung function does appear to be worthy of further study.

Although bronchiectatic patients as a group show very little change in lung function over many years ${ }^{13}$ it is possible that changes occur in a subset of such patients, and this should be considered in future studies.

In summary, we have studied the secretions of patients with bronchiectasis and found that elastase activity is a feature of purulent secretions and is associated with evidence of increased protein transudation into the lung secretions and a greater degree of air trapping. Whether these factors are important determinants of disease progression and whether they are susceptible to therapeutic modification are subjects of further studies.

The authors would like to thank Miss J Downs for typing the manuscript and Bencard for financial assistance. SLH was initially supported by the West Midlands Regional Health Authority.

\section{References}

'Stockley RA. Proteolytic enzymes, their inhibitors and lung disease. Clin Sci 1983;64:119-26.

2 Tegner H, Ohlsson K, Toremalm NG, Von Mecklenberg C. Effect of human leukocyte enzymes on tracheal mucosa and its mucociliary activity. Rhinology 1979;17: 199-206.

${ }^{3}$ Thurlbeck WM. Chronic airflow obstruction in lung disease. Philadelphia: WB Saunders, 1976:68-73.

${ }^{4}$ Laurenço RV, Loddenkemper R, Carton RW. Patterns of distribution and clearance of aerosols in patients with bronchiectasis. Am Rev Respir Dis 1972;106: 857-66.

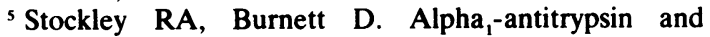
leukocyte elastase in infected and non infected sputum. Am Rev Respir Dis 1979;120:1081-6.

- Jackson AH, Hill SL, Afford SC, Stockley RA. Studies of sputum sol phase proteins and elastase activity in patients with cystic fibrosis. Eur $J$ Respir Dis 1984; 65:114-24.

' Hill SL, Stockley RA. Bronchiectasis-infection free or purulent free? Clin Sci 1982;62:38-9P (abstract).

${ }^{8}$ Stockley RA, Mistry M, Bradwell AR, Burnett D. A study of plasma proteins in the sol phase of sputum from patients with chronic bronchitis. Thorax 1979;34:777-82.

' Cotes JE. Lung function: assessment and application in medicine. 3rd ed. Oxford: Blackwell Scientific Publications, 1975:386-7.

${ }^{10}$ Lieberman J, Kaneshiro W. Inhibition of leucocyte elas- 
tase of purulent sputum by $\alpha_{1}$ antitrypsin. $J$ Lab Clin Med 1972;80:88-101.

"Twumasi DY, Liener IE. Proteases from purulent sputum: purification and properties of the elastase and chymotrypsin like enzymes. $J$ Biol Chem 1977;252:1917-26.

12 Wiggins J, Elliott JA, Stevenson RD, Stockley RA. Effect of corticosteroids on sputum sol-phase protease inhibitors in chronic obstructive pulmonary disease. Thorax 1982;37:652-6.

${ }^{13}$ Cherniak NS, Carton RW. Factors associated with respiratory insufficiency in bronchiectasis. Am J Med 1966;41:562-71.

14 Pande JW, Jain BP, Gupta RG, Guleria JS. Pulmonary ventilation and gas exchange in bronchiectasis. Thorax 1971;26:727-33. 